\title{
Intrinsically Flame Retardant Polyurethane Prepared with Epoxidized Soybean Oil and Vinylphosphonic Acid
}

\author{
Aynur Özşeker, Kemal Karadeniz, ${ }^{*}$ Raşit Fikret Yılmaz
}

\author{
Department of Chemistry, Faculty of Science and Literature, Sakarya University, Esentepe Campus, Serdivan, 54187, Sakarya, Turkey \\ * Corresponding author's e-mail address: kkaradeniz@sakarya.edu.tr
}

RECEIVED: July 11, 2018 * REVISED: January 03, 2019 * ACCEPTED: January 17, 2019

\begin{abstract}
The synthesis of novel polyol was carried out by the reaction of epoxidized soy-bean oil (ESBO) throught ring opening reaction with vinyl phosphonic acid (VPA) bearing two functional groups, vinyl and phosphonic acid. The synthesized polyol (PolP) and 4,4'- methylenebis(phenyl isocyanate) (MDI) were reacted to prepare biobased and inherently flame retardant polyurethane foam (PPPU). FTIR, ${ }^{1} \mathrm{H},{ }^{13} \mathrm{C}$ and ${ }^{31} \mathrm{P}$ NMR spectroscopy confirmed that the epoxy ring of ESBO was opened by phosphonic acid group of VPA, and vinyl group was not involved in the ring opening. The prepared polyurethane was characterized using fourier transformed infrared spectroscopy ( FTIR) and thermal properties were measured using differential scanning calorimetry (DSC) and thermogravimetric analysis (TGA). XRD was used for crystallografic structure and SEM image of polyurethane showed smooth surface. When compared to phosphorous-free polyurethanes derived from soybean oil based polyols, PPUP exibited better flame retardant property with a limiting oxygen value index of 26.4 .
\end{abstract}

Keywords: biopolymers, intrinsically flame retardance, foaming, polyurethanes.

\section{INTRODUCTION}

$\mathbf{R}$ EGULATIONS for buildings regarding fire retardancy and concerns over fossil fuel based resources intensified the studies on materials derived from biobased and flame retardant polymers. ${ }^{[1,2]}$ The researches are being focused on making the biopolymers be cost competitive with fossil fuel based polymers. ${ }^{[3-6]}$ The most used biopolymers are polyurethanes produced from polyols that are reaction product of diisocyanates and polyols synthesized by modification of plant oils. ${ }^{[7-13]}$ Plant oils such as soybean, corn, sunflower and palm oils are the most employed vegetable and renewable resources for the termosetting polyurethanes because of their abundancy, low cost and low toxicity. ${ }^{[14,15]}$ Vegetable oils are converted to polyol in many ways, a polyhydroxy compound which is one of the constituents of polyurethanes and polyesters that are useful in wide range of applications such as adhesives, sealents, foams, elastomers etc. ${ }^{[16,17]}$ Vegetable oils are functionalized over double bonds epoxidation first and followed by epoxy ring opening, hydrogenation of epoxide group, oxidation to aldehyde and then reduction to alcohol, esterification and transesterification of ester carbonyl group. ${ }^{[18-21]}$

Among the oils mentioned above, soybean oils have been intensively studied for the synthesis of polyols with epoxidation of double bounds utilizing peroxyacetic acid generated in situ from the reaction of glacial acetic acid and hydrogen peroxide, in the presence of an ion exchange catalyst. ${ }^{[22,23]}$ Epoxy ring opening reaction is used to introduce one or more hydroxyl group into fatty acid chain of epoxidized soybean oil (ESBO) that can be provided commercially at reasonable cost. Acid anhydrides, halogenated acids, thioethers and others, both mono or difunctional alcohols, amines, carboxylic acids, all of which bear one type of functional group have been reported as effective agents in the ring opening step. ${ }^{[24-26]}$

Ring opening reactions were also reported in our previous work using agents bearing two diffrent functional groups such as thio glycolic acid and glycolic acid.[27] One drawback of soy-based polyurethane like the other petrolbased polyurethanes is to be restricted to non-burning 
commercial applications due to its flammability. To make a polyurethane flame retardant, a flame retardant compound such as phosphorous or halogen groups are introduced in the polymer extrinsically or intrinsically. ${ }^{[28,29]}$ Polyurethanes generated from reactive flame-retardant monomer, either polyol or isocyanate exhibit more excellent flame-retardant properties than those with additive flame retardants. ${ }^{[30-32]}$

In our study, vinylphosphonic acid (VPA) bearing two different functional groups, vinyl and phosphonic acid, was used for ring opening of epoxidized soybean oil and a novel flame-retardant polyol which is named PolP was synthesized. Disappearance of epoxy bonds and formation of hydroxyl groups were confirmed using FTIR, NMR spectroscopy and MALDI-MS spectra. Differential Scanning Calorimetry (DSC) and thermogravimetric analysis were performed for the investigation of thermal behavior. Gel Permeation Chromatography (GPC) was used for the determination of molecular weights. PolP, and 4,4' methylenebis(phenyl isocyanate) (MDI) were used for rigid polyurethane foam. The polyurethane was characterized using FTIR, DSC, X-ray diffractometer and thermogravimetric analysis were done. The flame retardancy of PPPU was investigated using limiting oxygen index (LOI) testing. The thermal degradation of PPPU was investigated using thermogravimetric analysis (TGA). The apparent density of the foam was measured according to ASTM D1622-93. ASTM D1621 - 16 test method was used to measure compressive strength of the foam.

\section{EXPERIMENTAL}

\section{Materials}

Vinyl phosphonic acid (97\%) and 4,4'-methylenebis (phenyl isocyanate) were purchased from Merck KGaA, Germany, sodium sulfate anhydride, epoxidized soybean oil and sodium bicarbonate were purchased from Sigma-Aldrich. All these chemicals were used without further purification.

\section{Synthesis of Polyol P Using ESBO and VPA}

Phosphor containing Polyol (PolP) was synthesized by modification of the procedure previously reported in the literature. ${ }^{[27]} 12 \mathrm{~g}(0.013 \mathrm{~mol})$ of epoxidized soybeanoil, and $7.021 \mathrm{~g}(0.065 \mathrm{~mol})$ vinylphosphonic acid were mixed in a three necked, $100 \mathrm{ml}$, round-bottomed flask equipped with a thermometer, a stirrer and a reflux condenser and heated to $50^{\circ} \mathrm{C}$ under nitrogen atmosphere. After homogenization of reaction mixture for 15 minutes, mixing was allowed for $12 \mathrm{hrs}$ at $50^{\circ} \mathrm{C}$ for completion. The solution was washed with deionized water until $\mathrm{pH}: 7$ to remove unreacted vinyl phosphonic acid after the reaction mixture was coooled down to room temperature. The organic phase was

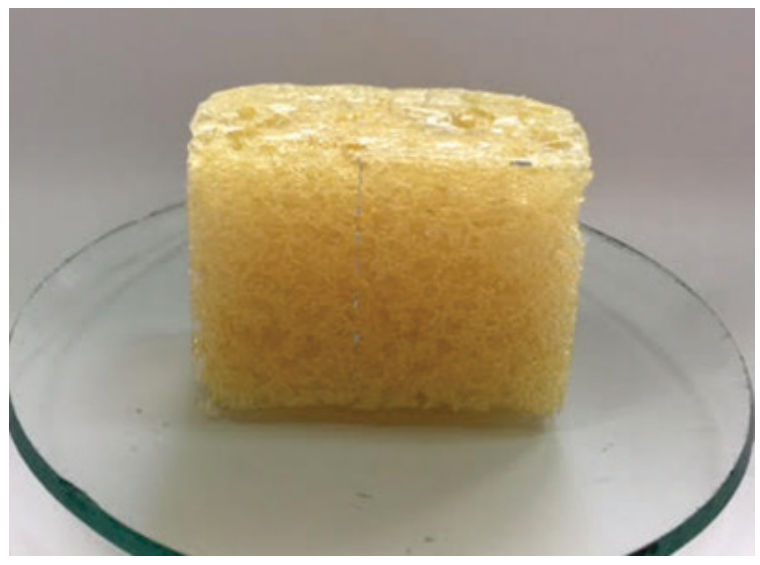

Figure 1. The Picture of polyurethane foam.

seperated first and dried with anhydrous sodium sulfate and solvent was evaporated at high vacum. A viscous yellow paled liquid was obtained with $91.7 \%$ yield. The hydroxyl value and acid value for PolP were $173.17 \mathrm{mg}$ $\mathrm{KOH} / \mathrm{g}$ and $2.02 \mathrm{mg} \mathrm{KOH} / \mathrm{g}$, respectively.

\section{Preparation of Polyurethane Foam}

One-shot hand mixing process was used to prepare polyurethane foam by mixing PolP with 4,4'methylenebis(phenyl isocynate) (MDI) at room temperature. Calculated amount of MDI starting from $200 \mathrm{mg}$ to $500 \mathrm{mg}$ was melted in a clean glass plate at $70{ }^{\circ} \mathrm{C}$ and hand mixed with $1000 \mathrm{mg}$ of PolP and $15 \mathrm{mg}$ of deionized water for 5 minutes, and then left for free rise. foam was formed gradualy (Figure 1). The required amount of MDI based on $\mathrm{OH}$ functionality of polyol was calculated as follows:

Mole of $\mathrm{OH}=$ Weight of polyol $(\mathrm{g}) \times \mathrm{OH}$ functionality

Mole of NCO $=$ Weight of MDI $(g) \times$ number of isocyanate group in $1.0 \mathrm{~g}$ of $\mathrm{MDI}$

The optimum $\mathrm{NCO} / \mathrm{OH}$ molar ratio for foam was 1.20/3.54. 0.30 g of MDI (\# of NCO group is 1.20) was used for $1.00 \mathrm{~g}$ of PolP (\# of OH group is 3.54). Rigidity of foam increased with increasing molar ratio. FTIR was also used to monitor the required amount of $\mathrm{MDI}$ by observing the disappearence of isocyanate peak at $2245-2268 \mathrm{~cm}^{-1}$.

\section{Characterization Techniques}

Shimadzu IR prestige-21 spectrophotometer equipped with ATR device, in a scanning range of $4000-650 \mathrm{~cm}^{-1}$ for 25 scans at a spectral resolution of $4 \mathrm{~cm}^{-1}$ was used for FTIR study. Data were collected and processed using IR solution software. Shimadzu Prestige-21 (200VCE) spectrophotometer operating at $300 \mathrm{MHz}$ and $75 \mathrm{MHz}$, respectively was used for ${ }^{1} \mathrm{H},{ }^{13} \mathrm{C}$ NMR and ${ }^{31}$ PNMR study. VNMR $6.1 \mathrm{C}$ software was used for data processing. Deuterated chloroform $\left(\mathrm{CDCl}_{3}, 99.8\right.$ atom \% D) at $15 \%$ concentration by volume was used for solutions. 


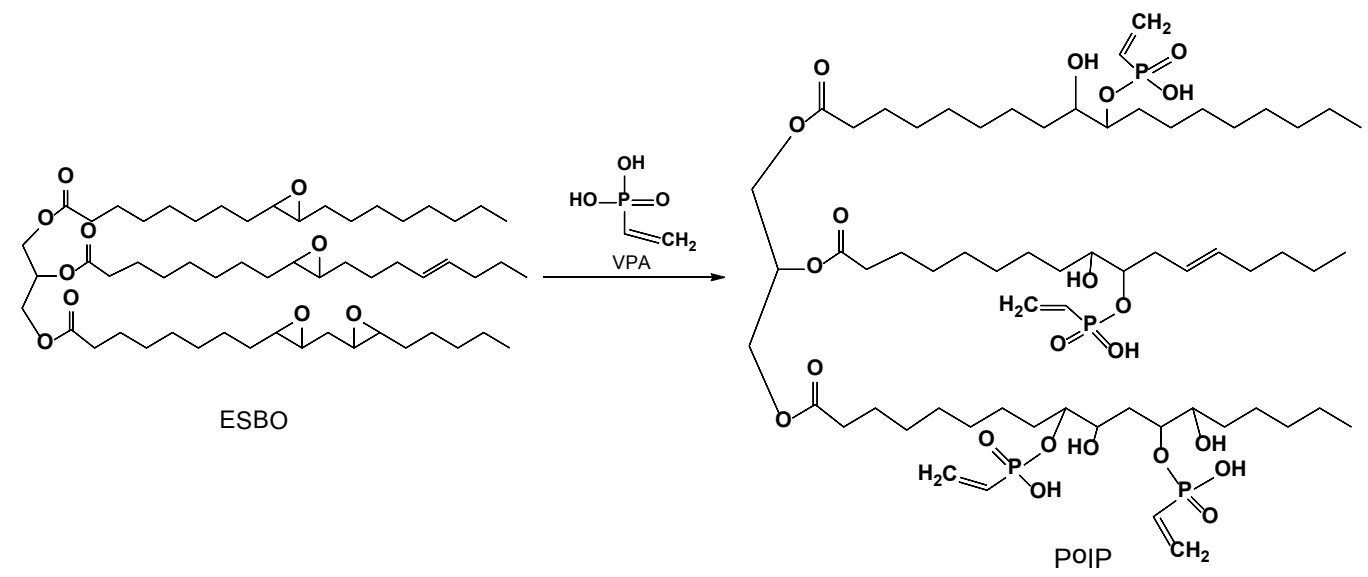

Figure 2. Scheme for the polyol formation fromthe reaction of ESBO with vinylphosphonic acid.

Mettler Toledo DSC1 200W with aluminium pans (40 $\mu \mathrm{L})$ and conducting cooling-heating cycles from $-90^{\circ} \mathrm{C}$ to 25 ${ }^{\circ} \mathrm{C}$ at a heating rate of $10^{\circ} \mathrm{C} \mathrm{min}^{-1}$ under nitrogen with 50 $\mathrm{mL} \mathrm{min}^{-1}$ flow rate was used for DSC measurements. Thermogravimetric analysis was conducted on Netzsch STA 44F1A analyzer with aluminium crucibles $(0.3 \mathrm{ml}, 40-60 \mathrm{mg}$ sample) by heating in nitrogen atmosphere $(20 \mathrm{~mL} / \mathrm{min})$ at a rate of $10^{\circ} \mathrm{C} / \mathrm{min}$ from 40 to $600^{\circ} \mathrm{C}$.

ASTM D-1957-86 was conducted for hydroxyl number determination and acid value was measured acording to ASTM D-4662. ASTM D 1652-04. Dynamic viscosity measurement of polyol was carried out using SV10 Series Sine-wave Vibro Viscometer of N\&D Company. Molecular weight of polyol was measured using Shimadzu Prominence GPC system equipped with a RID-10A refractive index detector, a LC- 20AD solvent delivery unit, a CTO-10AS column oven and a set of two columns, PSS SDV $5 \mu \mathrm{L} 1000 \AA \AA$ and PSS SDV $5 \mu \mathrm{L} 50 \AA$. An X-ray diffractometer (XRD-Rigaku D/MAX 2000) with CU-K radiation was used to measure crystaline properties. Compressive strength of the foam was measured on a Shimadzu AG-IC 20KN/50KN tensile machine using $56 \mathrm{~mm}$ long, $56 \mathrm{~mm}$ width, $25.4 \mathrm{~mm}$ thick samples at the compression rate of $2.5 \mathrm{~mm} / \mathrm{min}$. Limiting oxygen index (LOI) test was performed according to the ASTM D2863 - 13 using MARES Limiting oxygen tester, M LOI 01 . The dimensions of test specimens used were $100 \times 10 \times 10 \mathrm{~mm}$ in length $\times$ width $\times$ thickness.

\section{RESULTS AND DISCUSSION}

The synthesis of a novel soybean-based polyol obtained by epoxy ring opening using VPA bearing two different functionality, vinyl and phosphonic acid and then the preparation of phosphorous-containing polyurethane were achieved. The reaction route of ring opening reaction of ESBO with VPA can explained as shown in Figure 2. As the figure indicates, ring was opened by phosphonic acid group of VPA while vinyl group was not involved.

Figure 3 shows the synthesis scheme of polyurethane prepared by the reaction of PolP and MDI.

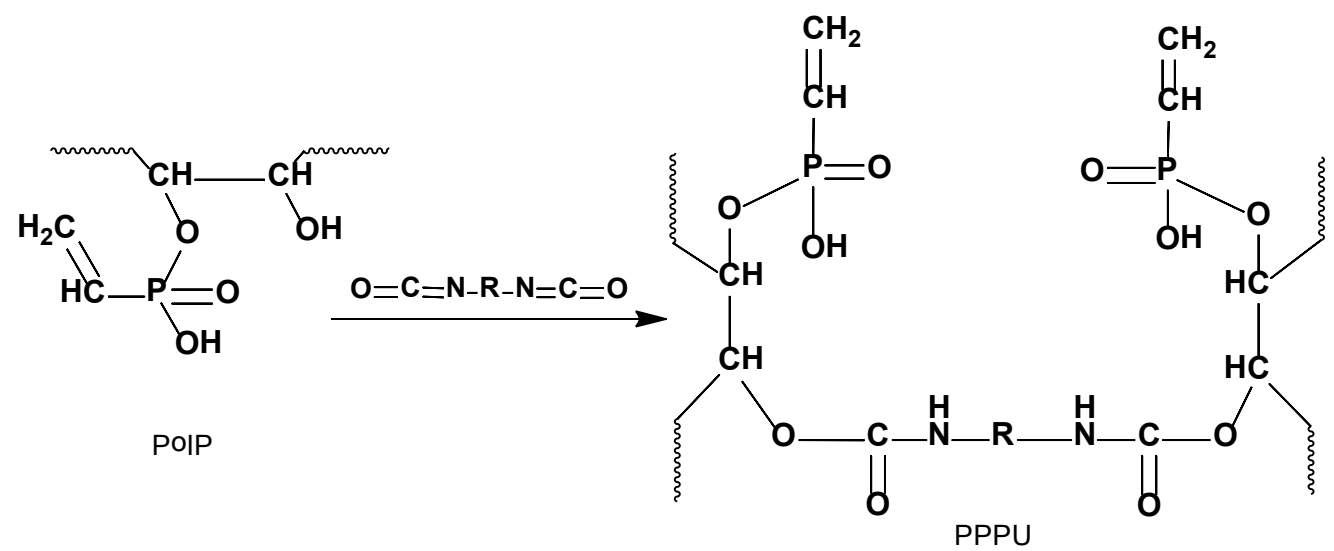

Figure 3. Proposed scheme for PPPU synthesis from PoIP and MDI. 


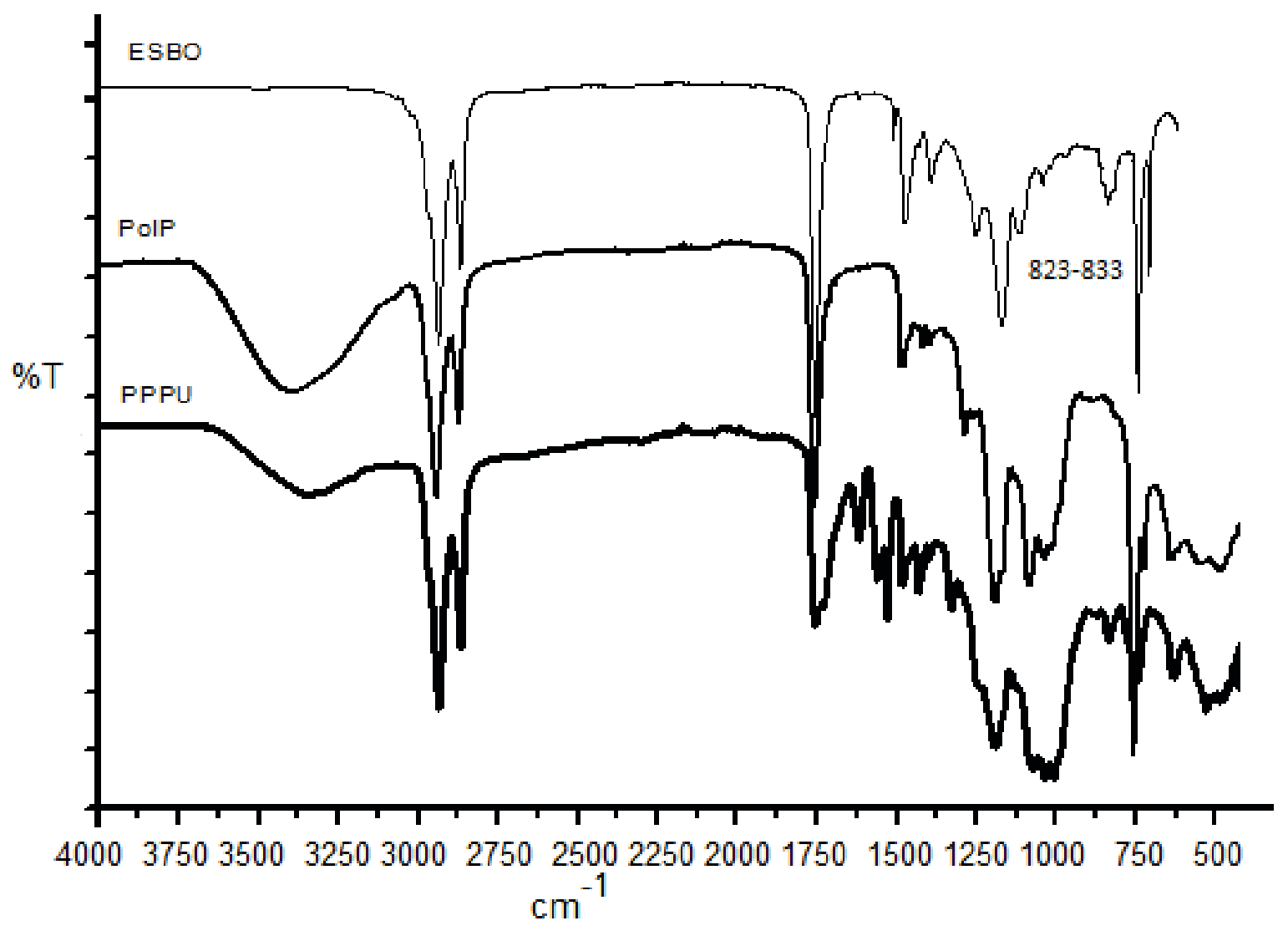

Figure 4. FTIR spectra of ESBO, PoIP and PPPU.

\section{Spectroscopic Analysis}

Disappearence of characteristic peaks of ESBO at 823 and $833 \mathrm{~cm}^{-1}$ and appearance of hydroxyl peaks at $3360 \mathrm{~cm}^{-1}$ confirmed that epoxy ring was opened and conversion of ESBO to polyol took place. The another evidence of polyol formation is that the peaks appeared at $1058 \mathrm{~cm}^{-1}$ and $1172 \mathrm{~cm}^{-1}$ could be attributed to $\mathrm{P}-\mathrm{O}-\mathrm{C}$ and $\mathrm{P}=\mathrm{O}$ frequencies respectively. Figure 4 shows the spectra of ESBO and PolP.

The peak at $1745 \mathrm{~cm}^{-1}$ seen at FTIR spectra of polyurethane and polyol can be attributed to carbonyl strectching vibrations. The peaks at 1525 and 1609 confirm the presence of $-\mathrm{NHCOO}-$ groups. The bands around 1058 and $1172 \mathrm{~cm}^{-1}$ are due to $\mathrm{P}-\mathrm{O}-\mathrm{C}$ and $\mathrm{P}=\mathrm{O}$ stretching frequencies respectively. ${ }^{[33]}$ These peaks can be seen in Figure 4.

Epoxy ring opening of ESBO with VPA was confirmed by appearance of $\mathrm{OH}$ proton at $5.2 \mathrm{ppm}$ in the ${ }^{1} \mathrm{H}$ NMR spectrum of PolP. Proton peaks of ESBO at $0.9-1.3 \mathrm{ppm}$ and at $1.5-2.3 \mathrm{ppm}$ can be attributed to terminal $-\mathrm{CH}_{3}$ groups and saturated $-\mathrm{CH}_{2}$ groups respectively. The signals at $3.4-3.9 \mathrm{ppm}$ can be attributed to methynic hydrogen- $\mathrm{CH}-\mathrm{OH}$ also confirmed the existence of bounded phosphanate groups. From the existence of the peak seen at $5.3 \mathrm{ppm}$ corresponding to methine protons, we can conlude that some double bonds remained unreacted (Figure 5). As for ${ }^{13} \mathrm{C}$ NMR spectra of ESBO and PolP, new peak in the spectra of PolP at $56 \mathrm{ppm}$ were due to $-\mathrm{CH}-$ carbons adjacent to hydroxyl groups. Disappearence of $\mathrm{CH}$ peaks at $64 \mathrm{ppm}$ and $70 \mathrm{ppm}$ confirmed the ring opening. In the ${ }^{31} \mathrm{P}$ NMR spectrum of PolP, the peak of the mono ester $\left[\mathrm{CH}_{2}=\mathrm{CH}-\mathrm{P}(\mathrm{O})(\mathrm{OH})(\mathrm{OR})\right]$ observed at $15.9 \mathrm{ppm}$ verified incorporation of phosphorous into polyol. ${ }^{[34]}$ The peak at $33.2 \mathrm{ppm}$ can be attributed to unreacted VPA $\left[\mathrm{CH}_{2}=\mathrm{CH}-\mathrm{P}(\mathrm{O})(\mathrm{OH})(\mathrm{OH}] .{ }^{[35]}\right.$

\section{GPC Analysis}

The gel permeation chromatography (GPC) was used to determine the values of number average molecular masses $\left(M_{\mathrm{n}}\right)$ and weight average molecular masses $\left(M_{\mathrm{w}}\right) . M_{\mathrm{n}}$ values were found for PolP as follows: 5724 (3.31 \%, $13.4 \mathrm{~min})$, 2722 (4.10\%, $13.9 \mathrm{~min}), 1393$ (68.88\%, $15.4 \mathrm{~min}), 975$ (19.13\%, $16.2 \mathrm{~min}), 458$ (2.23\%, $17.4 \mathrm{~min}), 342$ (2.34\%, 18 min) Weight average molecular weight $\left(M_{\mathrm{w}}\right)$, number 

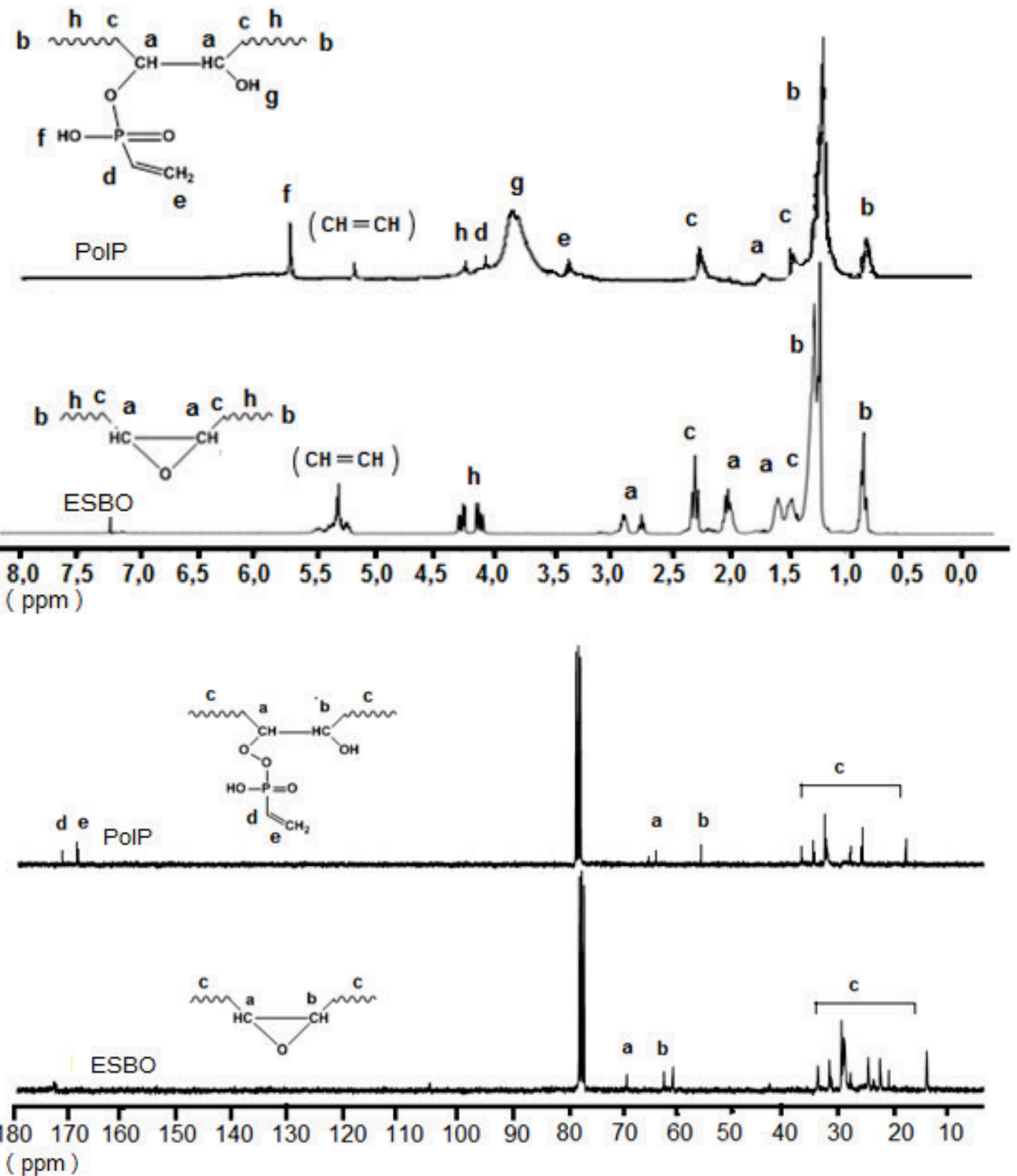

( ppm)

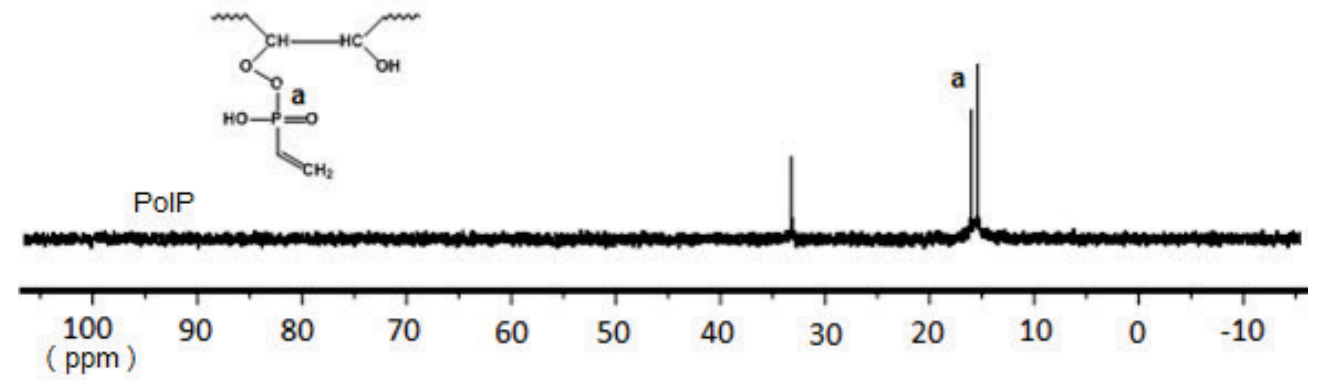

Figure 5. ${ }^{1} \mathrm{HNMR},{ }^{13} \mathrm{CNMR}$ and ${ }^{31} \mathrm{PNMRspectra}$ (top tobottom) of ESBO and PolP. 
Table 1. $M_{w}, M_{n}, \mathrm{PDI}, \mathrm{OH}$ number and acid number of pol $\mathrm{P}$

\begin{tabular}{|c|c|c|c|c|c|c|}
\hline Polyol & $M_{w}$ & $M_{n}$ & $\begin{array}{l}\text { PDI } \\
\left(M_{\mathrm{w}} / M_{\mathrm{n}}\right) \\
\end{array}$ & $\%$ of total & $\begin{array}{l}\text { OH Number } \\
\text { (mg KOH/g) }\end{array}$ & $\begin{array}{l}\text { Acid number } \\
\text { (mg KOH/g) }\end{array}$ \\
\hline ESBO & & & & & & 0.8 \\
\hline \multirow[t]{3}{*}{ PolP } & 5786 & 5724 & 1.01 & 3.31 & 173.74 & 2.02 \\
\hline & 2746 & 2722 & 1.03 & 4.10 & & \\
\hline & 1407 & 1393 & 1.01 & 68.8 & & \\
\hline
\end{tabular}

average molecular weight $\left(M_{\mathrm{n}}\right)$ and polydispersity index $\left(M_{\mathrm{w}} / M_{\mathrm{n}}\right)$ of PolP and can be seen in Table 1. Figure 6 shows the GPC chromatograms of ESBO and PoIP. High molecular weight products formed indicate that some side reactions occurred, causing the polyol to have complex structures and wide molecular weight distribution. The weak peaks beside the dominant peaks were due to mainly oligomerization, that is, the formation of dimers and trimers through the epoxy ring opening polymerization and chain coupling.

\section{Hydroxyl and Acid Value Measurements}

Acid value analysis was performed and the value was calculated using the following equation in $\mathrm{mg}$ of $\mathrm{KOH} / \mathrm{g}$ :

$$
\mathrm{AV}=\frac{V \times M \times 56.1}{m}
$$

Where, $V$ is the volume and $M$ is the molarity of the $\mathrm{KOH}$ solution and $m$ is the mass of the sample.

Hydroxyl value of PolP was measured $173.4 \mathrm{mg}$ of $\mathrm{KOH} / \mathrm{g}$ corresponding to $\mathrm{OH}$ functionality of 3.10 using the following equation:

$$
\mathrm{OHN}=\frac{B+\left(\frac{m A}{c}\right)-V}{m} M \times 56.1
$$

where, $B$ is the volume of $\mathrm{KOH}$ solution required for blank

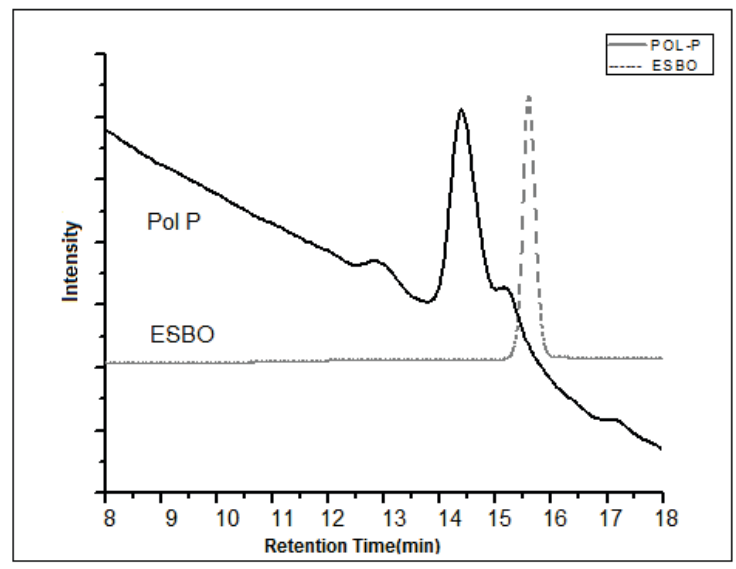

Figure 6. GPC chromatograms of ESBO and PoIP. reagent, $A$ is the $\mathrm{KOH}$ solution for titration of the acid value, $C$ is the mass of sample used for acid value, $V$ is the volume of the $\mathrm{KOH}$ solution for sample and $\mathrm{m}$ is the mass of the sample. The hydroxyl and acid values of ESBO and PoIP are presented in Table 1. It was seen from the table that the medium level of $\mathrm{OH}$ functionality (3.10) of PolP was sufficient to produce foam. Measured acid values of ESBO and PolP, 1.21and 2.02 respectively indicate the presence of some free acid in ESBO and the generation of free fatty acids and carboxylic acids during the reaction.

\section{Viscosity Measurement}

Quiet higher viscosity of PolP compared to ESBO, causing high molecular weight indicates that the formation of hydrogen bonding between newly generated hydroxyl groups and dimers or trimers resulting from some degree of oligomerisation. High molecular weight of PolP measured by GPC confirmed this ring opening oligomerization (Table 2).

\section{Differential Scanning Calorimetry Analysis}

DSC thermogram of PolP shows two melting points at $-44^{\circ} \mathrm{C}$ and $-25^{\circ} \mathrm{C}$, two crystaline points at $-8{ }^{\circ} \mathrm{C}$ and $0{ }^{\circ} \mathrm{C}$ indicating the crystalline structure (Figure 7). The thermograms suggest that the freezing points of PolP shifted to higher temperatures compared to the ESBO (Table 3). This can be interpreted by increasing inhomogenity resulting from intramolecular interaction of $\mathrm{OH}$ groups of polyol. No melting or crystallization is observed indicating the amorphous nature of PPPU, which is in agreement with XRD pattern of of polyurethane. PPPU shows a broad glass transition temperature at around $56^{\circ} \mathrm{C}$.

\section{Thermogravimetric Analysis}

Polyurethanes are known to be relatively unstable polymers. Vegetable -oil based polyurethanes exibit

Table 2. Dynamic viscosity data of ESBO and PolP at $250{ }^{\circ} \mathrm{C}$

\begin{tabular}{lc}
\hline Samples & Dynamic viscosity (mPa s) \\
\hline ESBO & 38 \\
Pol P & 8700 \\
\hline
\end{tabular}




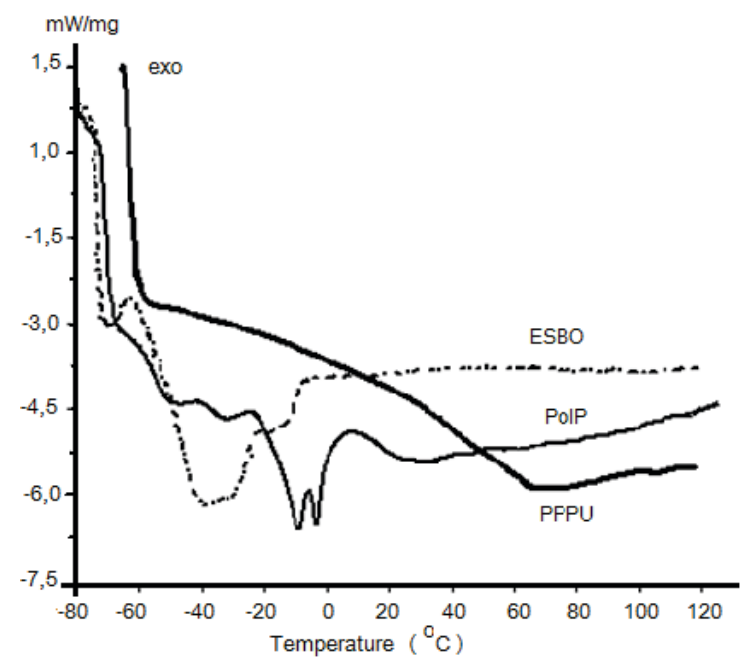

Figure 7. Differential scanning calorimetry thermograms of ESBO, PolP and PPPU.

relatively two or three degradation steps, mainly due to the presence of urethane bonds.[36] The Figure 8 shows TG curve and corresponding DTA degradation steps. First decomposition step at temperatures above $190{ }^{\circ} \mathrm{C}$ can be attributed to the decomposition of weak urethane bonds. This decomposition takes place through the dissociation to isocyanate and alcohol, the formation of primary amines and olefins, or the formation of secondary amines. The second decomposition temperature taking place at 381.3 ${ }^{\circ} \mathrm{C}$ was mostly due to decomposition of other segments of polymer networks. The gasification of any remaining components starts above $480^{\circ} \mathrm{C}$.

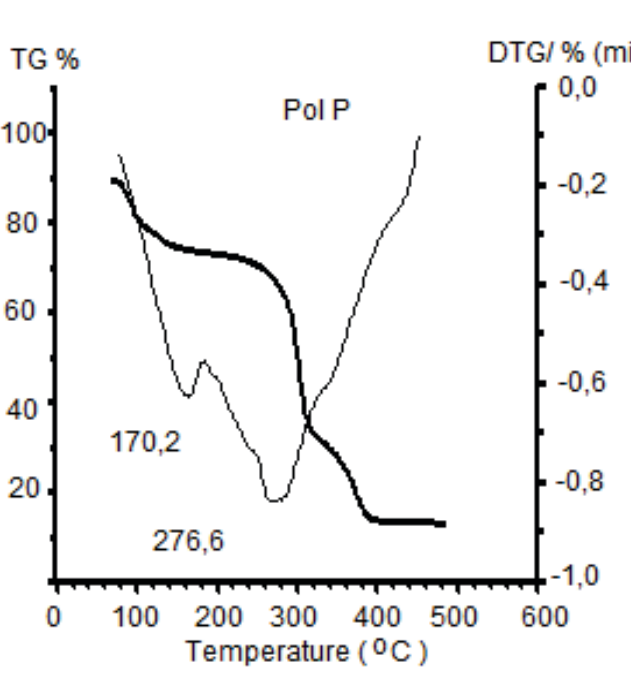

Figure 8. TG and DTG curves of polyol and polyurethane.
Table 3. Crystallization and melting temperatures of ESBO and PolP

\begin{tabular}{lll}
\hline & Temperature $/{ }^{\circ} \mathrm{C}$ & \\
& ESBO & PolP \\
\hline Peak 1 & -64 & -44 \\
Peak 2 & -38 & -25 \\
Peak 3 & -16 & -8 \\
Peak 4 & & 0 \\
\hline
\end{tabular}

\section{X-Ray Diffraction Analysis (XRD)}

The X-Ray diffraction analysis is used to determine the complexation and structure of polymer. As the Figure 9 shows, no crystalinity was observed and amorphous shoulder around $2 \Theta=15$ exists.

\section{Scanning Electron Microscopy and Ener- gy Dispersive X-Ray Analysis (SEM/EDX)}

Scanning electron microscopy and energy dispersive X-Ray analysis (SEM / EDS) were performed in order to see the surface morphology and elemental analysis of the polyurethane. Figure 10 displays the image of the sample and it can be seen that, some micro-sized particles exist on the surfaces of polyurethane. The presence of some degree of non-homogenity may be attributed to the presence of soft and hard segments. Elemental analysis of the PU sample confirmed that $\mathrm{P}$ atom was incorporated into polymer structure. The results of analysis are shown in Table 4.

\section{Flame retardant behavior of PolPPU}

The flame retardant behavior of polyurethane was characterized using LOI test which determines the 


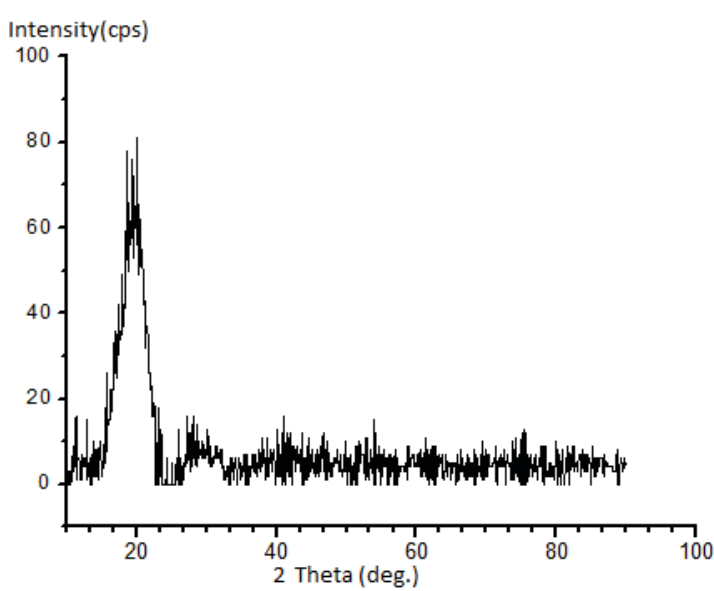

Figure 9. X-ray diffraction of polyurethane.

minimum oxygen concentration that maintains combustion of polymer. It was found that incorporation of phosphorous into polymer chain enhanced the flame retardant behavior of polyurethane and UL 94 standards were satisfied with $26.4 \%$ of LOI value. For the comparison, the flame retardancy of the soybean oil based three phosphorousfree polyurethanes that we reported in our previous work ${ }^{[28]}$ were measured and found much lower values with $21.8,20.9$ and $19.2 \%$ of LOI.

\section{Foam Density and Compression Strength}

Foam properties of polyurethane sample prepared by mixing PolP and MDI only, without any foam making additives were measured and considerable values obtained. Foam density and compressive strength were 90 $\mathrm{kg} / \mathrm{m}^{3}$ and $843 \mathrm{kPa}$ at $10 \%$ compression respectively.

\section{CONCLUSIONS}

Epoxy ring of epoxidized soybean oil was opened for the first time by the esterification reaction with vinyl phosphonic acid bearing vinyl and acid functionality. FTIR and NMR spectra indicated that phosphorous was incorporated into structure via O-P linkage. Synthesized novel PolP derived from soybean oil can be considered as a compatible flame-retardant polyol for polyuretane foam. Thermogravimetric analysis showed that the decomposition of foam started at $340{ }^{\circ} \mathrm{C}$ and gasification begins at $460{ }^{\circ} \mathrm{C}$. Compared to the non-phosphorous polyurethane, LOI value of PPPU increased to $26.4 \%$. It can be concluded that the polyurethane foam prepared from PolP has a considerable fire resistance even though it has $8 \%$ phosphorous only.

Conflicts of Interest. The authors declare that they have no conflicts of interest.

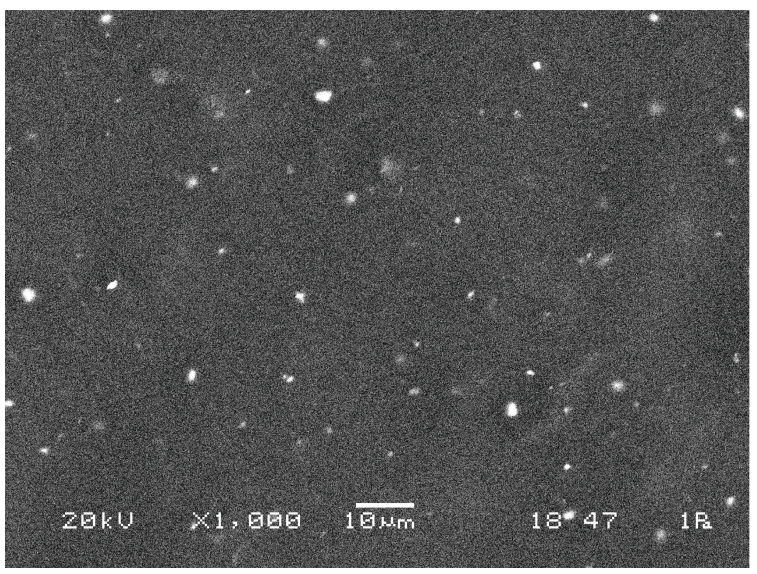

Figure 10. SEM micrograph of PPPU.

Funding Statement. This study was funded by The Research Fund of Sakarya University (BAP) (Project no: 2016-02-04046)

Acknowledgment. The authors acknowledge the financial support of The Research Fund of Sakarya University.

\section{REFERENCES}

[1] J. J. Bozell, M. Patel, ACS Symposium Series 921. American Chemical Society: Washington, DC, 2006.

[2] L. Chen, Y. Z. Wang, Polym. Advan. Technol. 2010, 21, 1.

[3] H. Miyagawa, M. Mishra, L. T. Drzal, A. K. Mohanty, Polymer 2005, 46, 445.

[4] F. Habib, M. Bajpai, Chem. Chem. Technol. 2011, 3, 317.

[5] Z. Petrovic, W. Zhang, I. Javni, Biomacromolecules 2005, 6, 713.

[6] G. Lligadas, J. C. Ronda, M. Galia, U. Biermann, J. O. Metzger, J. Polym. Sci. A 2006, 44, 634.

[7] K. S. Suresh, V. S. Kishanprasad, Ind. Eng. Chem. Res. 2005, 44, 4504.

[8] Y. Li, X. Luo, S. Hu, Bio-based Polyols and Polyurethanes, Springer, 2015, chapter 2, p. 15.

[9] A. Campanella, M. L. Bonnaillie, R. P. Wool, J. Appl. Polym. Sci. 2009, 112, 2567.

[10] S. Miao, S. Zhang, Z. Su, P. Wang, J. Polym. Sci. A 2010, 48, 243.

[11] Z. S. Petrovic, Polym. Rev. 2008, 48, 109.

[12] A. Campanella, L. M. Bonnaillie, R. P. Wool, J. Appl. Polym. Sci. 2009, 112, 2567.

[13] J. Jin, Q. Dong, Z. Shu, W. Wang, K. He, Procedia Eng. 2014, 71, 304.

[14] M. A. R. Meier, J. O. Metzger, U. S. Schubert, Chem. Soc. Rev. 2007, 36, 1788. 
[15] J. Zhang, J. J. Tang, J. X. Zhang, Int. J. Polym. Sci. 2015, 1.

[16] M. Desroches, M. Escouvols, R. Auvergne, S. Caillol, B. Boutevin, Polym. Rev. 2012, 52, 38.

[17] G. Lligadas, J. C. Ronda, M. Galia, V. Cadiz, Biomacromolecules 2010, 11, 2825.

[18] L. M. Espinosa, M.A. Meier, Eur. Polym. J. 2011, 47, 837.

[19] Z. S. Petrovic, L. Yang, A. Zlatanic, W. Zhang , I. Javni, J. Appl. Polym. Sci. 2007, 105, 2717.

[20] A. Kockritz, A. Martin, Eur. J. Lipid Sci. Technol. 2007, $110,812$.

[21] F. Stempfle, D. Quinzler, I. Heckler, S. Mecking, Macromolecules 2011, 44, 4159.

[22] L. Scala, R. P. Wool, J. Am. Oil Chem. Soc. 2002, 79, 373.

[23] S. J. Park, F. L. Jin, J. R. Lee, Macromol. Rapid. Comm. 2004, 25, 724.

[24] R. Chen, C. Zhang, M. R. Kessler, J. Appl. Polym. Sci. 2015, 132, 41213.

[25] A. Biswas, A. Adhvaryu, S. H. Gordon, S.Z. Erhan, J. L. Willet, J. Agric. Food Chem. 2005, 53, 9485.
[26] K. W. Lee, C. Hailan, J. Yinhua, Y. W. Kim, K. W. Chung, Korean J. Chem Eng. 2008, 25, 474.

[27] K. Karadeniz, H. Aki, M. Y. Sen, Y. Calikoglu, J. Am. Oil Chem. Soc. 2015, 92, 725.

[28] R. H. Patel, M. D. Shah, H. B. Patel, Int. J. Polym. Anal. Ch. 2011, 16, 107.

[29] M. Thirumal, D. Khastgir, G. B. Nando, Y. P. Naik, N. K.Singha, Polym. Degrad. Stab. 2010, 95, 1138.

[30] F. Çelebi, O. Polat, L. Aras, G. Gündüz, M. I. Akhmedov, J. Appl. Polym. Sci. 2004, 91, 1314.

[31] L. Zhang, M. Zhang, L. Hu, Y. Zhou, Ind. Crop. Prod. 2014, 52, 380.

[32] Q. Zhao, C. Chen, R. Fan, Y. Yuan, Y. Xing, X. Ma, J. Fire Sci. 2017, 35, 99.

[33] R. H. Patel, M. D. Shah, H. B. Patel, Int. J. Polym. Anal. Ch. 2011, 16, 107.

[34] M. Heinen, A. E. Gerbase, C. L. Petzhold, Polym. Degrad. Stab. 2014, 108, 76.

[35] H. Komber, V. Steinert, B. Voit, Macromolecules 2008, 41, 2119.

[36] L. B. Tavares, C. V. Boas, G. R. Schleder, A. M. Nacas, D. S. Rosa, D. J. Santos, Polym. Lett. 2016, 10, 927. 